\title{
Prevalência de obesidade em localidade do Estado de São Paulo, Brasil, 1987
}

\author{
Cecilia Amaro de Lolio*, Maria do Rosário Dias de Oliveira Latorre*
}

\begin{abstract}
LOLIO, C.A. de \& LATORRE, M. do R.D. de O. Prevalência de obesidade em localidade do Estado de São Paulo, Brasil, 1987. Rev. Saúde públ., S. Paulo, 25: 33-6, 1991. Foi estudada a prevalência de obesidade na população urbana de 18-74 anos residente em 1987 no Município de Araraquara, SP, Brasil, cidade média agroindustrial de 150.000 habitantes, situada a $250 \mathrm{~km}$ a noroeste da Capital do Estado. Foram estudados 1.126 habitantes, 502 do sexo masculino e 624 do feminino, selecionados por uma amostragem equiprobabilística por conglomerados. A prevalência de sobrepeso (Quetelet $25-29,9 \mathrm{~kg} / \mathrm{m}^{2}$ ) foi de $26,9 \%$ para o sexo masculino e de $27,7 \%$ para o feminino. A prevalência de obesidade (Quetelet maior ou igual a $30,0 \mathrm{~kg} / \mathrm{m}^{2}$ ) foi de $10,2 \%$ para o sexo masculino e de $14,7 \%$ para o feminino. Estas percentagens são bastante elevadas quando se as compara com as de países afluentes anglo-saxões. Discutem-se as causas do fenômeno, já que a cidade é um Município afluente de economia agroindustrial. Quando se definiram critérios próprios para Araraquara (P85 e P95 do Quetelet para a idade de 20-29 anos por sexo) verificou-se que o padrão "magro" para o Município é mais magro do que o similar norte-americano, o que torna criticável a extensão de critérios de corte oriundos de outras localidades para regiões de características diferentes.
\end{abstract}

Descritores: Obesidade, epidemiologia. Peso corporal.

\section{Introduçăo}

Araraquara é um município de 150.000 habitantes, localizado a $250 \mathrm{~km}$ a noroeste da Capital do Estado de São Paulo, com economia fortemente centrada na agroindústria do suco de laranja, cana de açúcar e milho. O Município representa, para fins deste estudo, o interior urbano do Estado de São Paulo, já que se trata de uma cidade sede de Região Administrativa, de tamanho médio. Para o ano de 1987, descreveu-se uma prevalência de hipertensão arterial em adultos da zona urbana que foi acentuadamente alta, a maior de quantas foram estudadas no Brasil ${ }^{3}$. Cerca de $28 \%$ da população urbana era hipertensa, usando-se como critério de definição o da Organização Mundial da Saúde. Por ocasião do citado estudo de prevalência de hipertensão arterial, outras variáveis, dentre elas o peso e a altura, foram também registradas, o que permite avaliar a prevalência da obesidade na mesma população.

Sabe-se que a obesidade, definida como uma proporção anormalmente elevada de gordura corpórea, é importante fator de risco para a hipertensão arterial sistêmica ${ }^{5}$. Nos últimos anos tem-se também discutido a importância do padrão de distribuição da gordura como precursor da hipertensão arterial $^{6}$. A maioria dos estudos de obe-

* Departamento de Epidemiologia da Faculdade de Saúde Pública da Universidade de São Paulo - Av. Dr. Amaldo, 715 - 01255 - São Paulo, SP - Brasil. sidade, contudo, atém-se a definir a obesidade como um aumento no peso corpóreo acima de um padrão arbitrário definido com relação à altura ${ }^{1,2}$, já que inexistem padrões de normalidade para 0 peso.

Assim, para julgar se uma pessoa é obesa ou não, algum tipo de indicador que correlacione peso e altura é utilizado. A forma de fazer isto varia: desde as tabelas de pesos ideais, segundo a estatura, para os quais existe menor risco de vida (estatísticas de companhias de seguros) até relaçōes entre peso e altura ${ }^{1,5}$ como por exemplo, 0 índice de massa corporal (Quetelet $=$ peso/ altura ${ }^{2}$ ) e o indice ponderal (altura/peso ${ }^{1 / 3}$ ). A correlação destes dois últimos índices com a gordura medida a partir da densidade corpórea é de 0,7 a 0,8 , sendo que o Quetelet é o que mais se correlaciona com a gordura corporea 2,4 .

Os estudos de obesidade também diferenciam o sobrepeso (overweight) da obesidade. No primeiro, o peso corporal situa-se entre o limite superior do normal e $20 \%$ acima deste limite. Já na obesidade, o peso situa-se acima de $20 \%$ do limite superior para a altura de tabelas como a de Fogarty ${ }^{1}$. Ao ser usada uma relação como o Quetelet para o diagnóstico de sobrepeso e obesidade, há dois grandes critérios:

- aquele utilizado pelos inquéritos da população geral norte-americana ${ }^{4}$ : estabelecem-se como níveis de corte o percentil 85 (P85) do Quetelet da população de 20-29 anos do mesmo sexo para o sobrepeso; e o percenti 95 (P95) do mesmo índice 
e da mesma população para a obesidade. A população referida é considerada como de compleição magra e qualquer acréscimo de peso é atribuído ao acúmulo de gordura. Em 1976-1980, tais níveis de corte eram: P85 masculino $=27,8 \mathrm{~kg} / \mathrm{m}^{2}$ e feminino $27,3 \mathrm{~kg} / \mathrm{m}^{2}$; P95: masculino maior ou igual a 31,1 $\mathrm{kg} / \mathrm{m}^{2}$ e feminino maior ou igual a $32,3 \mathrm{~kg} / \mathrm{m}^{2}$.

- aquele utilizado por outros pesquisadores ${ }^{1,2,5}$, que definem arbitrariamente 0 sobrepeso como Quetelet entre 25,0 e $29,9 \mathrm{~kg} / \mathrm{m}^{2}$, e a obesidade como Quetelet maior ou igual a $30,0 \mathrm{~kg} / \mathrm{m}^{2}$, independentemente da idade e do sexo do entrevistado.

Tomando por base estas consideraçðes, achouse oportuno:

a) estimar as prevalências de obesidade e de sobrepeso para a população urbana adulta (18-74 anos) residente em Araraquara, segundo idade e sexo;

b) comparar as prevalências estimadas com as de outros países;

c) sugerir, com base no estudo da população de 20-29 anos, critérios de corte para a definição de sobrepeso e de obesidade para a população de Araraquara, SP.

\section{Metodologla}

Populaçäo de estudo. A população de estudo foi constituída pela população civil de 18 a 74 anos, residente na zona urbana do Município de Araraquara, SP, em 1987.

Plano amostral. O plano amostral foi o de amostragem equiprobabilistica por conglomerados em três etapas, sendo na primeira etapa sorteados os setores censitários, na segunda, os quarteirões, e, na terceira, os domicílios. Nos domicílios sorteados foram entrevistados todos os indivíduos do grupo etário de interesse que residiam no mesmo. Foram feitas até três visitas ao mesmo domicílio para evitar perdas.

O tamanho da amostra foi calculado estimandose uma prevalência de hipertensão arterial de $17,5 \%$. Como a prevalência estimada de sobrepeso e de obesidade (somadas) situa-se em torno de $40 \%$ para a população norte-americana ${ }^{1}$, não há perda de precisão para o estudo de obesidade.

Delineamento do estudo e variáveis. Por se buscar as estimativas de prevalência, foi feito um estudo transversal (cross-sectional). Para tanto, a população de estudo foi entrevistada com a aplicação de um questionário onde foram registrados, dentre outros dados:

Idade - em anos completos no momento da entrevista;

Sexo

Peso - medido em quilogramas, mediante o uso de balanças portáteis (ARNO), do tipo usado em banheiro. A capacidade da balança era de até 130 $\mathrm{kg}$, com mostrador subdividido em 500 e $1.000 \mathrm{~g}$. Foram usadas cinco balanças, e cada uma era colocada em local plano e tarada inicialmente. $O$ entrevistado, portando roupas leves e sem sapatos, era posicionado de frente para o mostrador da balança. As entrevistadoras foram treinadas a evitar erros de leitura da escala de medida do peso por leitura obliqua do mostrador, bem como por aproximações das leituras para final 0 .

O peso foi dado como "ignorado" no caso de pessoas em que não era possível obtê-lo (imobilizadas no leito, ou em cadeira de rodas, ou usando aparelhos gessados), tendo estes casos sido excluídos da análise.

Altura - A altura foi medida em centímetros usando-se um metro duplo (tal como na construção civil), aliado a um esquadro de $60^{\circ}$ que funcionou como a régua de um antropômetro improvisado. Para este fim, pedia-se ao entrevistado que descalçasse os sapatos e se encostasse a um batente de porta, mantendo-se ereto e com pernas e calcanhares juntos, os braços ao longo do corpo, 0 olhar para o infinito, sem hiperextensão da coluna. Quando, por algum motivo, o entrevistador não podia ter sua altura medida, a mesma era dada como "ignorada" e tais casos foram excluídos da análise.

Análise. Foi calculado o índice de massa corporal (Quetelet) para cada entrevistado, com base na relação peso/ altura ${ }^{1}$, expressando-se o peso em kg e a altura em $\mathrm{m}$.

A seguir foram definidos os critérios de corte para:

- sobrepeso: pessoas com Quetelet entre 25,0 e $29,9 \mathrm{~kg} / \mathrm{m}^{2}$

- obesidade: pessoas com Quetelet maior ou igual a $30,0 \mathrm{~kg} / \mathrm{m}^{2}$.

A fim de estudar-se critérios de corte próprios da população de Araraquara, foram também calculados os P85 e P95 da população de 20-29 anos de cada sexo.

Compararam-se as prevalências de sobrepeso e de obesidade encontradas para a população de estudo, segundo sexo, com dados publicados de outros países (Grã-Bretanha, Estados Unidos e Austrália).

\section{Resultados}

Foram estudados 1.138 indivíduos com idades compreendidas entre 18 e 74 anos. Foram excluídos da análise 12 indivíduos com peso e/ou altura ignorados, resultando uma população de estudo de 1.126 indivíduos, sendo 502 do sexo 
Tabela 1. Distribuiçấo da amostra da populaçăo de 18 a 74 anos de idade de Araraquara, SP, 1987, segundo sexo e idade.

\begin{tabular}{cccc}
\hline \multirow{2}{*}{$\begin{array}{c}c \\
\text { Idade }\end{array}$} & \multicolumn{2}{c}{ Sexo } & Total \\
(Anos) & Masc. & Fem. & \\
\hline $18-24$ & 109 & 115 & 224 \\
$25-34$ & 143 & 161 & 304 \\
$35-44$ & 81 & 113 & 194 \\
$45-54$ & 73 & 105 & 178 \\
$55-64$ & 65 & 85 & 150 \\
$65-74$ & 31 & 45 & 76 \\
\hline Total & 502 & 624 & 1.126 \\
\hline
\end{tabular}

masculino e 624 do feminino que se encontram distribuídos segundo sexo e idade na Tabela 1.

A Tabela 2 apresenta a prevalência de sobrepeso segundo idade e sexo para a população de estudo. Pode-se verificar que a prevalência é crescente com a idade para os homens até a faixa etária de 35 a 44 anos quando então passa a declinar. Para as mulheres a prevalência é sempre crescente ficando em tornc de $28 \%$ para a população de estudo.

A Tabela 3 apresenta a prevalência de obesidade segundo idade e sexo. Para o sexo feminino, a prevalência cresce com a idade até os 45-54 anos, quando, então, passa a decrescer. Para o sexo masculino, a prevalência de obesidade varia bastante de estrato para estrato, porém aqueles situados entre 45-64 anos são os de mais alta prevalência de obesidade. Para o conjunto da popu-
Tabela 2 Prevalência (\%) de sobrepeso* segundo idade e sexo na população de 18 a 74 anos residente $r$ a zona urbana do Municfpio de Araraquara, SP, em 1987.

\begin{tabular}{cccc}
\hline Idade & \multicolumn{2}{c}{ Sexo } & Total \\
(Anos) & Masc. & Fem. & \\
\hline & & & \\
$18-24$ & 8,3 & 15,7 & 12,1 \\
$25-34$ & 25,9 & 19,9 & 22,7 \\
$35-44$ & 38,3 & 31,0 & 34,0 \\
$45-54$ & 37,0 & 35,2 & 36,0 \\
$55-64$ & 33,8 & 38,8 & 36,7 \\
$65-74$ & 29,0 & 40,0 & 35,5 \\
\hline Total & 26,9 & 27,7 & 27,4 \\
\hline
\end{tabular}

- Sobrepeso: Quetelet 25,0 - 29,9 kg/m²

Tabela 3. Prevalencia (\%) de obesidade* segundo idade e sexo na populaçăo de 18 a 74 anos residente na zona urbana do Município de Araraquara, SP, em 1987.

\begin{tabular}{crrr}
\hline \multirow{4}{*}{$\begin{array}{c}c \\
\text { Idade } \\
\text { (Anos) }\end{array}$} & Masc. & Fem. & Total \\
& & & \\
\hline & 4,6 & 3,5 & 4,0 \\
$25-24$ & 11,2 & 7,5 & 9,2 \\
$35-44$ & 9,9 & 14,2 & 12,4 \\
$45-54$ & 15,1 & 26,7 & 21,9 \\
$55-64$ & 15,4 & 25,9 & 21,3 \\
$65-74$ & 3,2 & 22,2 & 14,5 \\
\hline Total & 10,2 & 14,7 & 12,7 \\
\hline
\end{tabular}

- Obesidade: Quetelet $\geq 30,0 \mathrm{~kg} / \mathrm{m}^{2}$

Tabela 4. Percentagem de pessoas com sobrepeso* $\theta$ obesidade** segundo sexo em diferentes localidades.

\begin{tabular}{|c|c|c|c|c|c|c|}
\hline \multicolumn{2}{|c|}{ Localidade/Ano } & \multirow{2}{*}{$\begin{array}{c}\text { Idade da } \\
\text { Popul. de } \\
\text { Estudo }\end{array}$} & \multicolumn{2}{|c|}{ Sobrepeso (\%) } & $\begin{array}{l}\text { Obes } \\
\text { Masc. }\end{array}$ & $\begin{array}{l}\text { (\%) } \\
\text { Fem. }\end{array}$ \\
\hline $\begin{array}{l}\text { Austrália } \\
\text { Grã-Bretanha } \\
\text { Estados Unidos } \\
\text { Araraquara, BR }\end{array}$ & $\begin{array}{l}-1980 \\
-1983 \\
-1983 \\
-1987\end{array}$ & & $\begin{array}{l}34 \\
34 \\
31 \\
27\end{array}$ & $\begin{array}{l}24 \\
24 \\
24 \\
28\end{array}$ & $\begin{array}{r}7 \\
6 \\
12 \\
10\end{array}$ & $\begin{array}{r}7 \\
8 \\
12 \\
15\end{array}$ \\
\hline
\end{tabular}

- Sobrepeso: Quetelet entre 25 e $29,9 \mathrm{~kg} / \mathrm{m}^{2}$

* Obesidade: Quetelet maior ou igual a $30,0 \mathrm{~kg} / \mathrm{m}^{2}$ Fonte: Bray' (1985), para outras localidades.

Tabela 5. P85 e P95 do Quetelet* da populaçâo de 20-29 anos residente na zona urbana de Araraquara segundo sexo em 1987.

\begin{tabular}{lrr}
\hline \multicolumn{1}{c}{ Percentis } & \multicolumn{2}{c}{ Sexo } \\
& Masculino & Feminino \\
\hline P85 (sobrepeso) & 26,4 & 26,0 \\
P95 (obesidade) & $\geq 31,5$ & 229,5
\end{tabular}

\footnotetext{
- Percentis expressos em $\mathrm{kg} / \mathrm{m}^{2}$
}

lação, a prevalência de obesidade cresce com a idade até a meia idade, quando passa então a decrescer.

A Tabela 4 compara a percentagem das pessoas com sobrepeso e obesidade segundo o sexo em diferentes localidades estudadas. Pode-se verificar que a observada em Araraquara é bastante elevada quando comparada a de outros locais, sobretudo com relação à obesidade.

A Tabela 5 apresenta os P85 e P95 da população de 20-29 anos segundo sexo. 
Quando se comparam estes indicadores para o nível de corte com aqueles usados na população norte-americana, vê-se que o padrão brasileiro de 20-29 anos é mais magro do que aquele norteamericano, já que os valores são menores.

\section{Discussão}

A prevalência de sobrepeso e de obesidade grave em Araraquara revelou-se bastante elevada, fato que merece maior investigação. Inexistem dados de renda per capita e de consumo de alimentos em geral e para o ano de 1987 em particular. O fato de Araraquara ser uma cidade média numa região do interior urbano afluente poderia explicar estes dados. A maneira pela qual isto poderia ocorrer seria por uma maior ingestão de alimentos per capita (dietas hipercalóricas) aliada possivelmente a um menor dispêndio de energia por menor exercício físico.

É preciso notar que até o momento encontraramse para a mesma população duas condições importantes como fatores de risco para as principais causas de morte do Município (doenças coronárias e acidentes vasculares cerebrais): tanto a prevalência de hipertensão arterial quanto a de obesidade são aí acentuadamente elevadas. Deve-se mencionar ainda que uma das explicações plausíveis para a maior prevalência de hipertensão arterial é justamente a maior prevalência de obesidade.

Os resultados obtidos corroboram a opinião geral de que a obesidade e o sobrepeso são características marcantes da população adulta a partir da meia idade. Pode-se notar ainda que o padrão "magro" é para Araraquara mais magro do que o similar norte-americano. Este é um problema comum ao se comparar indicadores de massa corporal obtidos em outras localidades aplicando-os a outras populações que não a de origem. Emerge, conseqüentemente, como necessidade, o desenvolvimento de critérios de corte próprios e mais adequados para descrever a realidade brasileira que se sabe ser bastante diferente da norte-americana. Mesmo os dados obtidos para Araraquara não são passiveis de extensão a todas regiōes urbanas do País visto que se trata de uma cidade média afluente.
LOLIO, C.A. de \& LATORRE, M. do R. D. de O. [Prevalence of obesity in a County town of S. Paulo State, Brazil, 1987]. Rev. Saúde públ., S. Paulo, 25: 33-6, 1991. The prevalence of obesity in Araraquara, a county of 150,000 inhabitants situated $250 \mathrm{~km}$ from S. Paulo, Brazil was studied. The study population, of 18-74 yrs. of age, resident in the urban area in 1987, was composed of 1,126 inhabitants, 502 males and 624 females, selected through a cluster equiprobabilistic sampling process. The prevalence of overweight (Quetelet $25-29.9 \mathrm{Kg} / \mathrm{m}^{2}$ ) was $26.9 \%$ for males and $27.7 \%$ for females. The prevalence of obesity (Quetelet equal or greater than $30.0 \mathrm{~kg} / \mathrm{m}^{2}$ ) was $10.2 \%$ for males and $14.7 \%$ for females. These percentages are high when compared with those of affluent Anglo-saxon countries. The reasons for this phenomenon are discussed in the light of the fact that the economy of the city is affluent and is dependent on agroindustrial activities. When own cul-off criteria (P85 and P95 for Quetelet for 20-29 yrs of age, for each sex) are calculated, the "lean pattern" for Araraquara County is leaner than that for the a United States population. This raises doubts as to the possibility of applying cut-off criteria of particular regions to regions of different characteristics.

Keywords: Obesity, epidemiology. Body weight.

\section{Referênclas Bibllográficas}

1. BRAY, G.A. Obesity: definition, diagnosis and disadvantages. Med. J. Australia, 142:S2-S8, 1985.

2. BRAY, G.A. Obesity: basic considerations and clinical approaches. Dis. - Mon., 35(7) July, 1989.

3. LOLIO, C.A. de Prevalência de hipertensão arterial no Município de Araraquara, SP, em 1987. São Paulo, 1989. [Tese de Doutorado "Faculdade de Medicina da USP].

4. NAJJAR, M.F. \& ROWLAND, M. Anthropometric reference data and prevalence of overweight: United States, 1976-1980. Vital Hlth Statist. Ser. 11 (238), 1987.

5. PI-SUNIER, F.X. Obesity. In: Shils, M.E. \& Young, V.R., ed. Modern nutrition in health and disease. 7 th ed. Philadelphia, Lea \& Febiger, 1988. p. 795-816.

6. SELBY, J. V.; FRIEDMAN, G. D.; QUESENBERRY Jr., C.P. Precursors of essential hypertension: the role of body fat distribution pattem. Amer. J. Epidem., 129: 43-53, 1989.

\author{
Recebido para publicação em 20/4/1990 \\ Reapresentado em 23/11/1990 \\ Aprovado para publicação em 29/11/1990
}

